\title{
Breakable Solid Transportation Problem with Hybrid and Fuzzy Safety Factors using LINGO and Genetic Algorithm
}

Abhijit Baidya*, Uttam Kumar Bera and Manoranjan Maiti

Department of Mathematics, National Institute of Technology, Agartala, Jirania 799055, West Tripura, India

\begin{abstract}
In this paper we present a solution of solid transportation problem (STP) for breakable items with different environments. If we carrying the produce from sources to destination by the means of unlike conveyances then due to insurgency, land slide and bad road, there are some risks or difficulties to transport the items. By this motive we initiate "Safety Factors" in transportation problem. Due to this reason desired total safety factor is being introduced. Also our objective is to evaluate the solution of STP using expected value model. Here we develop six models where first three models are formulated taking crisp unit transportation cost but the remaining three models are formulated taking hybrid unit transportation cost. To build up the different models we consider breakability and safety factor which is taken as crisp, fuzzy and hybrid for assorted models. All the fuzzy and hybrid models are reduced into its crisp equivalent using expected value modeling. Finally by Generalized Reduced Gradient (GRG) method using LINGO.13 optimization software and Genetic Algorithm we solve the mathematical models and put a enlarge discussion on it.
\end{abstract}

Keywords: Solid transportation problem; Safety factor; Hybrid variables; Triangular fuzzy number; breakability; Expected value model; Genetic algorithm

\section{Introduction}

The transportation problem (TP) was developed by Hitchcock [1]. The classical transportation problem deals with transportation goods from some sources to some destinations. The solid transportation problem (STP) is a generalization of the well-known transportation problem (TP) in which three-dimensional properties is taken into account in the objective and constraint set instead of source and destination. The STP was first stated by Shell [2]. In many industrial problems, a homogeneous product is delivered from an origin to a destination by means of different modes of transport called conveyances, such as trucks, cargo flights, goods trains, ships, etc. These conveyances are taken as the third dimension. A solid transportation problem can be converted to a classical transportation problem by considering only a single type of conveyance. Transportation problems normally are formulated as arrangement problems in which carrying are made from sources to destinations as well as from destinations to destinations also. Sometimes there are restrictions on the flow of transportation. In the projected traditional transportation problem, transportation may be made from all sources to all destinations, if required and no transportation from destinations to destinations or from sources to sources is permissible. Also, the distances between the origins and destinations are not here taken into account as in the network problems. Actuality most of transportation problems are unbalanced for breakable items as the supplied amount by the suppliers (i.e., origins) is not equal to the received amount by the retailers (i.e., destinations). Few of these items are glass-goods, toys, ceramic goods, etc. Till now the materials of these type has not been considered for transportation models. Zadeh [3] first introduced the concept of fuzzy set theory. Later several authors such as Zadeh, Kaufmann, Zimmermann, Liu, Dubois and Prade [4-8] developed and applied fuzzy set theory. Chanas and Kuchta [9] studied transportation problem with fuzzy cost coefficients. [10] considered two types of uncertain STP, one with interval numbers and other with fuzzy numbers. Liu and Liu [11] presented expected value model for fuzzy programming. Yang and Liu [12] applied expected value model, chance-constrained programming model and dependent- chance programming in fixed charge solid transportation problem in fuzzy environment. Applied possibility programming approach to a material requirement planning problem with fuzzy constraints and fuzzy coefficients, using the definition of possibility measure of fuzzy number. Hybrid variable was first stated by Liu in 2006 but in 2009 $\mathrm{Li}$ and Liu proposed the expected value of hybrid variable. In some realistic transportation systems, transported amount from a source inversely depends on the level of unit transportation cost. When the unit transportation cost in a particular route is low, then a decision maker (DM) tries to transport the maximum amount of the item through that route, i.e., the transported amount is high. Again on the contrary, if the unit transportation cost is high, then less resource is transported. For medium unit transport cost, the transported amount is also medium. Chanas and Kuchta and Omar and Samir [13,14] discussed the solution algorithm for solving the transportation problem in fuzzy environment. Grzegorzewski and Chanas [15,16] approximated the fuzzy number to its nearest interval. Grzegorzewski and Mrowka [17] approximated a general fuzzy number to trapezoidal or triangular fuzzy numbers. Due to insufficient information, lack of confirmation and vary financial market, the available data of a transportation system such as resources, demands and conveyance capacities are not always crisp or precise but are fuzzy or stochastic or both. So the fuzziness and randomness can be present in the objective function as well as in the constraints of a STP. Dealing with different types of uncertainty in many practical problems is still an emerging problem. Recently Kundu et al. [18] solve a multi-objective solid transportation problem with

*Corresponding author: Abhijit Baidya, Department of Mathematics, National Institute of Technology, Agartala, Jirania 799055, West Tripura, India, Tel: 0381234 6360; E-mail: abhijitnita@yahoo.in

Received August 04, 2014; Accepted August 27, 2014; Published August 31 2014

Citation: Baidya A, Bera UK, Maiti M (2014) Breakable Solid Transportation Problem with Hybrid and Fuzzy Safety Factors using LINGO and Genetic Algorithm. J Appl Computat Math 3: 185. doi:10.4172/2168-9679.1000185

Copyright: $\odot 2014$ Baidya A, et al. This is an open-access article distributed under the terms of the Creative Commons Attribution License, which permits unrestricted use, distribution, and reproduction in any medium, provided the original author and source are credited. 
hybrid penalty cost. Also Baidya et al. [19] works on safety factor and uncertainty. Our aspire in this paper is to formulate and solve singleobjective solid transportation problem (SOSTP) with safety constraints with special types of uncertain (fuzzy and hybrid) parameters. The fixed charge problem was initialized by Hirsch in 1968. Up to now, it has been widely applied in many decision-making and optimization problems. Interested readers may refer to Kennington and Unger, Sun et al., Gottlieb and Paulmann [20-23], and so on. In this paper, we shall consider the fuzzy fixed charge STP. In spite of so many developments in literature, there are some lacunas in solid transportation problem and these are:

(i) Some researcher such as Chanas and Kuchta [13] studied transportation problem with fuzzy cost coefficients. Jimenez and Verdegay in 1999 [10] considered two types of uncertainty (interval and fuzzy) in solid transportation problem but nobody can solve any STP by taking hybrid uncertainty.

(ii) Very few STPs are available for breakable items; no STP model is formulated for hybrid and fuzzy safety factor and breakability.

In this paper, an item with breakability rate is transported from origins to destinations through dissimilar conveyances. We formulate six models without and with safety factor and breakability where these safety factor and breakability are crisp, fuzzy and hybrid. To organize this manuscript we employ the unit transportation cost as crisp number, fuzzy number and hybrid number and also to convert the uncertainty models into its crisp corresponding we apply expected value modeling. In some model decision maker (DM) likes to minimize the transportation cost choosing the particular routes and modes of transportation for particular so that total safety for the system is greater or equal to a predefined safety value. In rising countries, due to insurgency, all routes for transportation are not equally safe. Furthermore there are some risks of running some conveyances (modes) in some particular routes. In STP, none has taken this safety factor into account for development, through it is very much prevalent in different parts of India, including North-East region and Maoist dominated areas.

\section{Preliminaries}

\section{Definitions}

Fuzzy number: A fuzzy subset $\tilde{A}$ of real number with membership function is said to be a fuzzy number if

$\mu_{\tilde{A}}(\mathrm{x})$ is upper semi-continuous membership function;

$\widetilde{A}$ is normal, i.e., there exists an element $\mathrm{x}_{0}$ such that $\mu_{\widetilde{A}}\left(\mathrm{x}_{0}\right)$;

$\tilde{A}$ is fuzzy convex, i.e. $\mu_{\tilde{A}}\left(\lambda x_{1}+(1-\lambda) x_{2} \geq \mu_{\tilde{A}}\left(x_{1}\right) \wedge \mu_{\tilde{A}}\left(x_{2}\right) \forall \mathrm{x}_{1}, x_{2} \in R\right.$ and $\lambda \in[0,1]$

Support of $\tilde{A}=\left\{x \in R: \mu_{\tilde{A}}(\mathrm{x})>0\right\}$ is bounded.

Fuzzy numbers are represented by two types of membership functions: (a) Linear membership functions e.g. triangular fuzzy number (TFN), Trapezoidal fuzzy number, Piecewise Linear fuzzy number etc. (b) Non-linear membership functions e.g. Parabolic fuzzy number (PFN), Exponential fuzzy number and other non-linear fuzzy number. We used the following fuzzy numbers:

Triangular Fuzzy Number (TFN): Triangular Fuzzy Number (TFN) is the fuzzy number $\widetilde{A}=\left(\mathrm{a}_{1}, \mathrm{a}_{2}, \mathrm{a}_{3}\right)$ with the membership function $\mu_{\tilde{A}}(x)$

a continuous mapping: $\mu_{\tilde{A}}: R \rightarrow[0,1]$

$$
\mu_{\tilde{A}}(\mathrm{x})=\left\{\begin{array}{l}
0 \quad \text { for }-\infty<x<a_{1} \\
\frac{x-a_{1}}{a_{2}-a_{1}} \text { for } a_{1} \leq x<a_{2} \\
\frac{a_{3}-x}{a_{3}-a_{2}} \text { for } a_{2} \leq x \leq a_{3} \\
0 \quad \text { for } a_{2}<x<\infty
\end{array}\right.
$$

General Fuzzy Number (GFN): It is known that for any fuzzy number $\widetilde{A}$ there exist four numbers $a_{1}, a_{2}, a_{3}, a_{4} \in R$ and two functions $f(x), g(x): R \rightarrow[0,1]$ where $f(x)$ is non-decreasing and $g(x)$ is nonincreasing, such that we can describe a membership function $\mu_{\tilde{A}}(\mathrm{x})$ in a following manner (Figures 1 and 2)

$$
\mu_{\tilde{A}}(\mathrm{x})=\left\{\begin{array}{lc}
0 & \text { for } x<a_{1} \\
f(\mathrm{x}) & \text { for } \mathrm{a}_{1} \leq x<a_{2} \\
1 & \text { for } a_{2} \leq x \leq a_{3} \\
g(\mathrm{x}) & \text { for } \mathrm{a}_{3} \leq x \leq a_{4} \\
0 & \text { for } a_{4} \leq x \leq \infty
\end{array}\right.
$$

Credibility measure: Credibility measure was presented by Liu and Liu (2002). For a fuzzy variable $\xi$ with membership function $\mu_{\xi}(\mathrm{x})$ and for any set $\mathrm{B}$ of real numbers, credibility measure of fuzzy event $\{\xi \in \mathrm{B}\}$ is defined as

$$
C r\{\xi \in B\}=\frac{1}{2}(\operatorname{Pos}\{\xi \in B\}+N e c\{\xi \in B\}),
$$

where possibility and necessity measures of $\{\xi \in \mathrm{B}\}$ are respectively defined as

$$
\begin{aligned}
& \operatorname{Pos}\{\xi \in B\}=\sup _{\mathrm{X} \in B}{ }^{c} \mu_{\xi}(\mathrm{x}) \\
& \text { And } \\
& \operatorname{Nec}\{\xi \in B\}=1-\operatorname{Sup}_{X \in B}{ }^{c} \mu_{\xi}(\mathrm{x})
\end{aligned}
$$

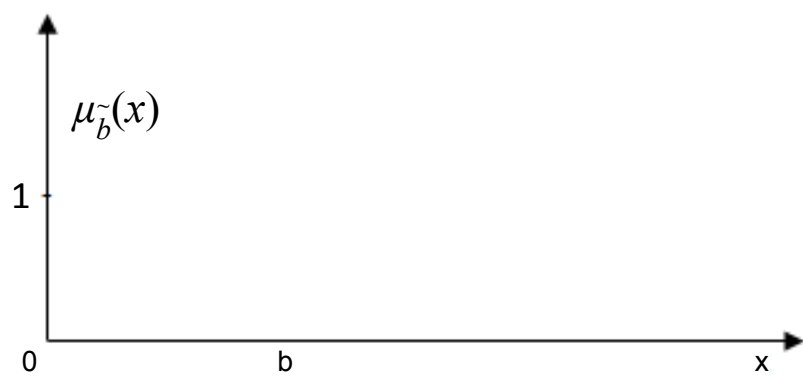

Figure 1: Membership function of a crisp number b.

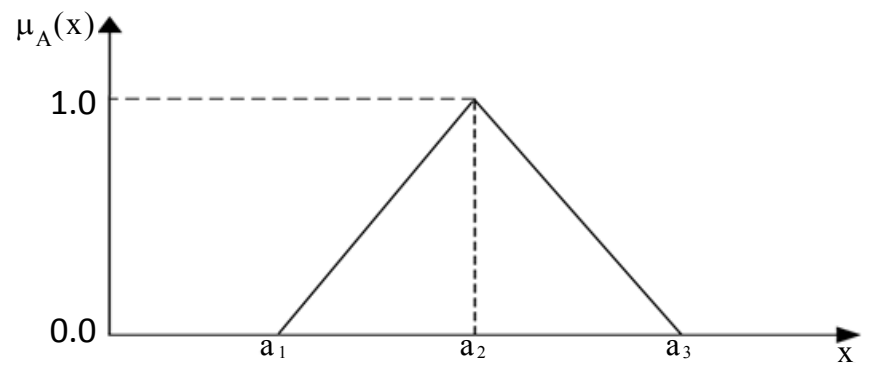

Figure 2: Triangular Fuzzy Number. 


\section{Expected value of fuzzy variable:}

Definition: Let $\xi$ be a fuzzy variable. Then the expected value of $\xi$ is defined by $E(\xi)=\int_{0}^{+\infty} C r\{\xi \geq \mathrm{r}\} \mathrm{dr}-\int_{-\infty}^{0} C r\{\xi \leq \mathrm{r}\} d r$

Provided that at least one of the two integrals is finite. The equipossible fuzzy variable on $[a, b]$ has an expected value $\frac{(a+b)}{2}$ The triangular fuzzy variable $(a, b, c)$ has an expected value $\frac{(a+2 b+c)}{4}$ The trapezoidal fuzzy variable $(a, b, c, d)$ has an expected value $\frac{(\mathrm{a}+\mathrm{b}+\mathrm{c}+\mathrm{d})}{4}$

Let $\xi$ be the continuous nonnegative fuzzy variable with membership function $\mu$ if $\mu$ is decreasing on

$$
E[\xi]=\frac{1}{2} \int_{0}^{\infty} \mu(\mathrm{x}) \mathrm{dx}
$$

Example-1: A fuzzy variable $\xi$ is called exponentially distributed if has an exponential membership function

$$
\mu(\mathrm{x})=2\left(1+\exp \left(\frac{\pi x}{\sqrt{6 m}}\right)\right)^{-1}, x \geq 0, m>0
$$

Then the expected value is $\frac{\sqrt{6 m} \text { in } 2}{\pi}$

Theorem-1: Let $\xi$ be a continuous fuzzy variable with membership function $\mu$ If its expected value exists, and there is a point $\mathrm{x} 0$ such that $\mu(\mathrm{x})$ is increasing on $\left(-\infty, \mathrm{x}_{0}\right)$ and decreasing on $\left(\mathrm{x}_{0},+\infty\right)$ then

$$
E[\xi]=\mathrm{x}_{0}+\frac{1}{2} \int_{x_{0}}^{+\infty} \mu(\mathrm{x}) \mathrm{dx}-\frac{1}{2} \int_{-\infty}^{x_{0}} \mu(\mathrm{x}) \mathrm{dx}
$$

Proof:

$$
\begin{aligned}
& \text { If } x_{o} \geq 0 \text {, then } \\
& C r\{\xi \leq \mathrm{r}\}=\left\{\begin{array}{c}
\frac{1}{2}[1+1-\mu(\mathrm{x})], \text { if } 0<\mathrm{r} \leq \mathrm{x}_{0} \\
\frac{1}{2} \mu(\mathrm{x}), \text { if } \mathrm{r}>\mathrm{x}_{0}
\end{array}\right. \\
& \text { And } C r\{\xi \leq \mathrm{r}\}=\left\{\frac{1}{2} \mu(\mathrm{x})\right. \\
& E[\xi]=\int_{0}^{x_{0}}\left[1-\frac{1}{2} \mu(\mathrm{x})\right] d x+\int_{x_{0}}^{+\infty}\left[\frac{1}{2} \mu(\mathrm{x})\right] d x- \\
& \int_{-\infty}^{0}\left[\frac{1}{2} \mu(\mathrm{x})\right] d x=x_{0}+\frac{1}{2} \int_{x_{0}}^{+\infty}\left[\frac{1}{2} \mu(\mathrm{x})\right] d x \\
& -\frac{1}{2} \int_{-\infty}^{x_{0}}\left[\frac{1}{2} \mu(\mathrm{x})\right] d x
\end{aligned}
$$

If $x_{0}<0$, a similar way may prove the equation (5). The theorem is proved. Especially, let $\mu(\mathrm{x})$ be symmetric function on the line $x=x_{o}$, then $E[\xi]=\mathrm{x}_{\mathrm{o}}$

Example-2: Let $\xi$ be triangular fuzzy variable (a, b, c), then it has an expected value

$$
\begin{aligned}
& E[\xi]=\mathrm{b}+\frac{1}{2} \int_{b}^{c} \frac{x-c}{b-c} d x-\frac{1}{2} \int_{b}^{c} \frac{x-a}{b-a} d x=b+\frac{c-b}{4}+ \\
& \frac{a-b}{4}=\frac{a+2 b+c}{4}
\end{aligned}
$$

Example-3: Let $\xi$ be equipossible fuzzy variable on [a, b], then it has an expected value

$$
E[\xi]=\frac{a+b}{2}+\frac{1}{2} \int_{\frac{a+b}{2}}^{b} 1 d x-\frac{1}{2} \int_{a}^{\frac{a+b}{2}} 1 d x=\frac{a+b}{2}
$$

Example-4: Let $\xi$ be trapezoidal fuzzy variable (a, b, c, d), then it has an expected value

$$
\begin{aligned}
& E[\xi]=\frac{b+c}{2}+\frac{1}{2} \int_{\frac{b+c}{2}}^{c} 1 d x+\frac{1}{2} \int_{c}^{d} \frac{x-d}{c-d} d x-\frac{1}{2} \int_{a}^{b} \frac{x-a}{b-a} d x \\
& -\frac{1}{2} \int_{b}^{\frac{b+c}{2}} 1 d x=\frac{a+b+c+d}{4}
\end{aligned}
$$

Especially, let $\xi$ be triangular fuzzy variable $(a, b, c)$ and $b-a=c-b$, then it has an expected value $E[\xi]=\mathrm{b}$. Let $\xi$ be trapezoidal fuzzy variable $(\mathrm{a}, \mathrm{b}, \mathrm{c}, \mathrm{d})$ and $\mathrm{b}-\mathrm{a}=\mathrm{d}-\mathrm{c}$, then it has an expected value $E[\xi]=\frac{b+c}{2}$

A fuzzy variable $\xi$ is called normally distributed if it has a normal membership function

$$
\mu(\mathrm{x})=2\left(1+\exp \left(\frac{\pi|x-e|}{\sqrt{6 \sigma}}\right)\right)^{-1}, x \in R, \sigma>1
$$

By theorem 1, the expected value is e.

The definition of expected value operation is also applicable to discrete case. Assume that $\xi$ be a simple fuzzy variable whose membership function is given by

$$
\mu(\mathrm{x})=\left\{\begin{array}{l}
\mu_{1}, \text { if } x=a_{1} \\
\mu_{2}, \text { if } x=a_{2} \\
\mu_{m}, \text { if } x=a_{m}
\end{array}\right.
$$

Where $\mathrm{a}_{1}, \mathrm{a}_{2}, \ldots \mathrm{a}_{\mathrm{m}}$ are distinct numbers. Note that Then $\mu_{1} V \mu_{2} V \mu_{3} V \ldots \ldots V \mu_{m}=1$ the expected value of is

$$
E[\xi]=\sum_{i=1}^{m} w_{i} a_{i}
$$

Where the weights are given by

$$
\begin{aligned}
& w_{i}=\frac{1}{2}\left(\max _{1 \leq \mathrm{j} \leq \mathrm{m}}\left\{\mu_{\mathrm{j}} \mid \mathrm{a}_{\mathrm{j}} \geq \mathrm{a}_{\mathrm{i}}\right\}-\right. \\
& \max _{1 \leq \mathrm{j} \leq \mathrm{m}}\left\{\mu_{\mathrm{j}} \mid \mathrm{a}_{\mathrm{j}}<\mathrm{a}_{\mathrm{i}}\right\}+\max _{1 \leq \mathrm{j} \leq \mathrm{m}}\left\{\mu_{\mathrm{j}} \mid \mathrm{a}_{\mathrm{j}} \geq \mathrm{a}_{\mathrm{i}}\right\}- \\
& \left.\max _{1 \leq \mathrm{j} \leq \mathrm{m}}\left\{\mu_{\mathrm{j}} \mid \mathrm{a}_{\mathrm{j}}>\mathrm{a}_{\mathrm{i}}\right\}\right)
\end{aligned}
$$

For $\mathrm{i}=1,2, \ldots . ., \mathrm{m}$. It is easy to verity that all and the sum of all $w_{i} \geq 0$ weights is just 1 .

Optimistic and pessimistic value: Let $\xi$ be a fuzzy variable and $\alpha \in[0,1]$. Then $\left.\xi_{\text {sup }}(\alpha)=\operatorname{SUP}\{\xi \geq r\} \geq \alpha\right\}$ is called $\alpha$ - optimistic value to $\xi$;

$$
\text { And }
$$

$\xi_{\text {inf }}(\alpha)=\operatorname{SUP}\{\mathrm{r}: \operatorname{cr}\{\xi \geq r\} \geq \alpha\}$ is called $\alpha-$ pessimistic value to

Random variable: For the probability space $(\Omega, \mathrm{S}, \mathrm{P})$ where $\Omega$ is a set of elementary events, $S$ is a set of all events (a $\sigma$-field of events) and $P: S \rightarrow[0,1]$ is a probability function, the mapping $\widehat{X}:(\Omega, \mathrm{S}, \mathrm{P}) \rightarrow \mathrm{R}$ is called a random variable. By the probability distribution function of the random variable $\widehat{X}$ we mean a function $F(\mathrm{x})=P\{\mathrm{w}: \widehat{\mathrm{X}}(\mathrm{w}) \leq \mathrm{x}\}$ for all $\mathrm{x} \in \mathrm{N}$ with $\mathrm{F}(-\infty)=0$ and $F(\infty)=1$

\section{Hybrid variable}

Definition: Suppose $(\Theta, P, \mathrm{Cr})$ is a credibility space $(\Omega, \mathrm{S}, \mathrm{Pr})$ and be a probability space. The product $(\Theta, P, \mathrm{Cr}) \mathrm{x}(\Omega, \mathrm{S}, \mathrm{Pr})$ is a chance space. 
Definition: Then a chance measure of an event $\wedge$ is defined as $\operatorname{ch}(\wedge)=$

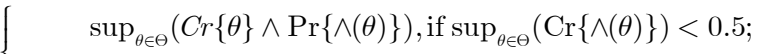

$$
\begin{aligned}
& \left\{1-\sup _{\theta \in \Theta}(C r\{\theta\} \wedge \operatorname{Pr}\{\wedge(\theta)\}), \text { if } \sup _{\theta \in \Theta}(\operatorname{Cr}\{\theta\} \wedge \operatorname{Pr}\{\wedge(\theta)\}) \geq 0.5\right. \text {; } \\
& \text { Then } \operatorname{ch}(\phi)=0, \operatorname{ch}\{\Theta \times \Omega\}=1,0 \leq \operatorname{ch}\{\wedge\} \leq
\end{aligned}
$$

1 For any event $\wedge$

Definition: A hybrid variable is a measurable function from a chance space $(\Theta, P, \operatorname{Cr}) \mathrm{x}(\Omega, \mathrm{S}, \operatorname{Pr})$ to real numbers, i.e. for any Borel set $\mathrm{B}$ of real numbers, the set $\{\xi \in \mathrm{B}\}=\{(\theta, \mathrm{w}) \in \Theta \times \Omega: \xi(\theta, \mathrm{w}) \in \mathrm{B}\}$ is an event.

Remark: A hybrid variable degenerates to a fuzzy variable if the value of

$\xi(\theta, \mathrm{w})$ does not vary with w. For example, $\xi(\theta, w)=\theta, \xi(\theta, w)=\theta 2+1, \xi(\theta, w)=\sin \theta$

Remark: A hybrid variable degenerate to a random variable if the value of $\xi(\theta, \mathrm{w})$ does not vary with $\theta$. For example, $\xi(\theta, w)=w, \xi(\theta, w)=w^{2}+1, \xi(\theta, w)=\sin w$

Remark: A hybrid variable $\xi(\theta, \mathrm{w})$ may also be regarded as a function from a credibility space $(\Theta, \mathrm{P}, \mathrm{Cr})$ to the set $\{\xi(\theta,) \mid. \theta \in \Theta\}$ random variable. Thus $\xi$ is a random fuzzy variable defined by Liu (2006).

Remark: A hybrid variable $\xi(\theta, \mathrm{w})$ may also be regarded as a function from a probability space $(\Omega, \mathrm{A}, \operatorname{Pr})$ to the set $\{\xi(., \mathrm{w}) \mid \mathrm{w} \in \Omega\}$ of fuzzy variables. If $\operatorname{Cr}\{\xi(., \mathrm{w}) \in \mathrm{B}\}$ is a measurable function of $w$ for any Borel set $\mathrm{B}$ of real number, then $\xi$ is a fuzzy random in the sense of Liu and Liu (2009) (Figure 3).

Example: If $\tilde{a}$ is a fuzzy variable and $\hat{n}$ is a random variable, then the $\operatorname{sum} \tilde{\xi}=\tilde{a}+\hat{n}$ is a hybrid variable, i.e. if $f: \Re^{2} \rightarrow \Re$ is a measurable function, then $\tilde{\xi}=f(\tilde{a}, \hat{n})$ is a hybrid variable. Now suppose that $\tilde{a}$ has a membership function $\mu$ and $n$ has a probability density function $\phi$ Then for any Borel set B of real numbers, we have

$$
\operatorname{ch}\{f(\tilde{\mathrm{a}}, \hat{\mathrm{n}}) \in \mathrm{B}\}=\left\{\begin{array}{c}
\sup _{x}\left[\frac{\mu(\mathrm{x})}{2} \wedge \int_{f(\mathrm{x}, \mathrm{y}) \mathrm{B}} \phi(\mathrm{y}) \mathrm{dy}\right], \text { if } \sup _{x}\left[\frac{\mu(\mathrm{x})}{2} \wedge \int_{f(\mathrm{x}, \mathrm{y}) \in \mathrm{B}} \phi(\mathrm{y}) \mathrm{dy}\right]<0.5 ; \\
1-\sup _{x} \sup _{x}\left[\frac{\mu(\mathrm{x})}{2} \wedge \int_{f(\mathrm{x}, \mathrm{y}) \mathrm{B}} \phi(\mathrm{y}) \mathrm{dy}\right], \text { if } \sup _{x}\left[\frac{\mu(\mathrm{x})}{2} \wedge \int_{f(\mathrm{x}, \mathrm{y}) \mathrm{B}} \phi(\mathrm{y}) \mathrm{dy}\right] \geq 0.5 ;
\end{array}\right.
$$

$\tilde{\varepsilon}$

$\approx$ Definition: Let $\hat{\xi}$ be a hybrid variable. Then the expected value of $\xi$ is defined by

$$
E[\tilde{\hat{\xi}}]=\int_{0}^{+\infty} \operatorname{ch}\{\tilde{\hat{\xi}} \geq r\} d r-\int_{-\infty}^{0} \operatorname{ch}\{\tilde{\hat{\xi}} \leq r\} d r \text { provided that at }
$$

least one of the integrals is finite.

Example: For the hybrid variable $\tilde{\hat{\xi}}=\tilde{a}+\hat{n}$, expected value of $\tilde{\hat{\xi}}$ is $E(\tilde{\hat{\xi}})=\mathrm{E}(\tilde{\mathrm{a}})+E(\hat{\mathrm{n}})$ For example, if $\tilde{a}=\left(\mathrm{t}_{1}, \mathrm{t}_{2}, \mathrm{t}_{3}\right)$ is

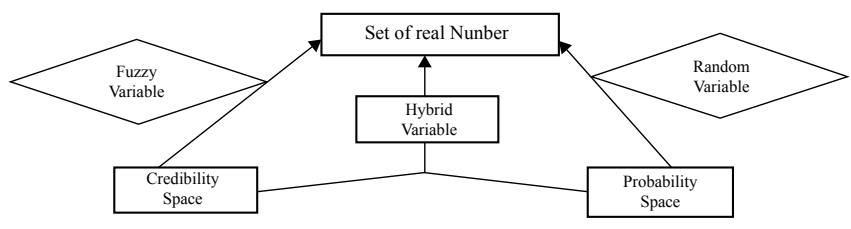

Figure 3: Geographical representation of Hybrid variable. triangular fuzzy number and $\hat{n}=N\left(\mu, \sigma^{2}\right)$ is normally distributed random variable. Then $E(\tilde{\hat{\xi}})=\frac{t_{1}+t_{2}+t_{3}}{4}+\mu$.

\section{Genetic Algorithm}

The Genetic algorithm (GA) was first proposed by Holland [23]. Genetic algorithm is a well-known computerized stochastic search method based on the evolutionary theory of Charles Darwin survival of the fittest and natural genetics (Goldberg, 1989). GA has successfully been applied to optimization problems in different fields, like engineering design, optimal control, transportation and assignment problems, job scheduling, inventory control and other reallife decision-making problems. The most fundamental idea of Genetic Algorithm is to imitate the natural evolution process artificially in which populations undergo continuous changes through genetic operators, like crossover, mutation and selection. Genetic algorithm can easily be implemented with the help of computer programming. In particular, it is very useful for solving complicated optimization problems which cannot be solved easily by direct or gradient based mathematical techniques. It is very effective to handle large-scale, reallife, discrete and continuous optimization problems without making unrealistic assumptions and approximations. Keeping the imitation of natural evolution as the foundation, genetic algorithm can be designed appropriately and modified to exploit special features of the problem to solve. This algorithm starts with an initial population of possible solutions (called individuals) to a given problem where each individual is represented using some form of encoding as a chromosome. These chromosomes are evaluated for their fitness. Based on their fitness, chromosomes in the population are to be selected for reproduction and selected individuals are manipulated by two known genetic operations, like crossover and mutation. The crossover operation is applied to create offspring from a pair of selected chromosomes. The mutation operation is used for a little modification/change to reproduce offspring. The repeated applications of genetic operators to the relatively fit chromosomes result in an increase in the average fitness of the population over generation and identification of improved solutions to the problem under investigation. This process is applied iteratively until the termination criterion is satisfied. The following functions and values are adopted in the proposed GA to solve the problem.

\section{Chromosome representation}

The concept of chromosome is normally used in the GA to stand for a feasible solution to the problem. A chromosome has the form of a string of genes that can take on some value from a specified search space. The specific chromosome representation varies based on the particular problem properties and requirements. Normally, there are two types of chromosome representation - (i) the binary vector representation based on bits and (ii) the real number representation. In this research work, the real number representation scheme is used.

Here, a "K dimensional real vector" $X=\left(\mathrm{x}_{1}, \mathrm{x}_{2}, \ldots, \mathrm{X}_{\mathrm{k}}\right)$ is used to represent a solution, where $\mathrm{x}_{1}, \mathrm{x}_{2}, \ldots, \mathrm{X}_{\mathrm{k}}$ represent different decision variables of the problem.

\section{Initialization}

A set of solutions (chromosomes) is called a population. $\mathrm{N}$ such solutions $\mathrm{x}_{1}, \mathrm{x}_{2}, \ldots, \mathrm{X}_{N}$ are randomly generated from search space by random number generator such that each $X_{i}$ satisfies the constraints of the problem. This solution set is taken as initial population and is the starting point for a GA to evolve to desired solutions. At this 
step, probability of crossover $P_{c}$ and probability of mutation $P_{m}$ are also initialized. These two parameters are used to select chromosomes from mating pool for genetic operations- crossover and mutation respectively.

\section{Fitness value}

All the chromosomes in the population are evaluated using a fitness function. This fitness value is a measure of whether the chromosome is suited for the environment under consideration. Chromosomes with higher fitness will receive larger probabilities of inheritance in subsequent generations, while chromosomes with low fitness will more likely be eliminated. The selection of a good and accurate fitness function is thus a key to the success of solving any problem quickly. In this thesis, value of a objective function due to the solution $\mathrm{X}$, is taken as fitness of $X$. Let it be $f(X)$.

\section{Selection process to create mating pool}

Selection in the GA is a scheme used to select some solutions from the population for mating pool. From this mating pool, pairs of individuals in the current generation are selected as parents to reproduce off spring. There are several selection schemes, such as roulette wheel selection, ranking selection, stochastic universal sampling selection, local selection, truncation selection, tournament selection, etc. Here, Roulette wheel selection process is used in different cases. This process consist of following steps-

(i) Find total fitness of the population $F=\sum_{i=1}^{N} f\left(\mathrm{x}_{\mathrm{i}}\right)$

(ii) Calculate the probability of section $p r$ of each solution by the formula $p r_{i}=\frac{f\left(\mathrm{x}_{\mathrm{i}}\right)}{F}$.

(iii) Calculate the cumulative probability $q r_{i}$ for each solution $X_{i}$ by the formula $q r_{i}=\sum_{j=0}^{i} p r_{j}$

(iv) Generate a random number " $r$ " from the range $[0,1]$.

(v) If $r<q r_{1}$ then select $X_{1}$ otherwise select $X_{i}(2 \leq \mathrm{i} \leq \mathrm{N})$ where $q r_{i-1} \leq r \leq q r_{i}$.

(vi) Repeat step (iv) and (v) $\mathrm{N}$ times to select $\mathrm{N}$ solutions from current population. Clearly one solution may be selected more than once.

(vii) Let us denote this selected solution set by $P^{1}(\mathrm{~T})$.

\section{Crossover}

Crossover is a key operator in the GA and is used to exchange the main characteristics of parent individuals and pass them on the children. It consists of two steps:

(i) Selection for crossover: For each solution $P^{1}(\mathrm{~T})$ generate a random number $\mathrm{r}$ from the range [0,1]. If $r<p_{c}$ then the solution is taken for crossover, where $p_{c}$ is the probability of crossover.

(ii) Crossover process: Crossover taken place on the selected solutions. For each pair of coupled solutions $Y_{1}, Y_{2}$ a random number $\mathrm{c}$ is generated from the range $[0,1]$ and $Y_{1}, Y_{2}$ are replaced by their offspring's $Y_{11}, Y_{21}$ respectively where $Y_{11}=c Y_{1}+(1=\mathrm{c}) \mathrm{Y}_{2}, Y 21=c Y 2+(1-\mathrm{c}) \mathrm{Y}_{1}$ provided $\quad Y_{11}, Y_{21}$ satisfied the constraints of the problem.

\section{Mutation}

The mutation operation is needed after the crossover operation to maintain population diversity and recover possible loss of some good characteristics. It is also consist of two steps:

(i) Selection for mutation: For each solution of $P^{1}(\mathrm{~T})$ generate a random number $r$ from the range [0,1]. If $r<p_{m}$ then the solution is taken for mutation, where $p_{m}$ is the probability of mutation.

(ii) Mutation process: To mutate a solution $X=\left(X_{1}, \mathrm{X}_{2}, \ldots, \mathrm{X}_{K}\right)$ select a random integer $\mathrm{r}$ in the range $[1 . . \mathrm{K}]$. Then replace $x_{r}$ by randomly generated value within the boundary of rth component of $\mathrm{X}$. Following selection, crossover and mutation, the new population is ready for its next iteration, i.e., $P^{1}(\mathrm{~T})$ is taken as population of new generation. With these genetic operations a simple genetic algorithm takes the following form. In the algorithm $\mathrm{T}$ is iteration counter, $\mathrm{P}(\mathrm{T})$ is the population of potential solutions for iteration $\mathrm{T}$, evaluate fitness of each members of $P(\mathrm{~T})$

\section{GA Algorithm}

1. Set iteration counter $\mathrm{T}=0$. $p_{m}$

2. Initialize probability of crossover $p_{c}$ and probability of mutation

3. Initialize $\mathrm{P}(\mathrm{T})$.

4. Evaluate $\mathrm{P}(\mathrm{T})$.

5. Repeat

a. Select $\mathrm{N}$ solutions from $\mathrm{P}(\mathrm{T})$, for mating pool using Roulettewheel selection process. Let this set be $\mathrm{P}(\mathrm{T}) 1$.

b. Select solutions from $\mathrm{P}(\mathrm{T}) 1$, for crossover depending on $p_{c}$

c. Made crossover on selected solutions for crossover to get population $\mathrm{P}(\mathrm{T}) 2$.

d. Select solutions from $\mathrm{P}(\mathrm{T}) 2$, for mutation depending on $p_{m}$

e. Made mutation on selected solutions for mutation to get population $\mathrm{P}(\mathrm{T}+1)$.

f. $\mathrm{T} \leftarrow \mathrm{T}+1$

g. Evaluate $\mathrm{P}(\mathrm{T})$.

6. Until (Termination condition does not hold).

7. Output: Fittest solution (chromosome) of $\mathrm{P}(\mathrm{T})$.

\section{Constraints Handling in GA}

The main idea of handling constraints is to design chromosomes carefully by genetic operators to keep all these within the feasible solution set. To ensure that the chromosomes (solutions) are feasible, we have to check all the new chromosomes ( $\mathrm{x}$ ) generated by genetic operators. We suggest that a function is designed for each target optimization problem, the output value 1 means that the chromosome is feasible, 0 for infeasible. The algorithm for finding the feasibility of an individual (solution) (x) for the optimization problem (2.60) is as follows:

for $\mathrm{j}=1$ to $l$ do

if $\left(g_{j}(X) \leq 0\right)$ continue; else return 0 ; endif endfor

for $\mathrm{k}=1$ to $\mathrm{m}$ do 
if $\left(\mathrm{h}_{\mathrm{k}}(\mathrm{x})=0\right)$ continue; else return 0 ; endif endfor return 1

\section{Description of the Problem}

STP is a problem of transporting goods from some sources to some customers through some conveyances (modes of transportation) and the main objective is to find optimal transportation plan so that the total transportation cost is minimum. Also the goods transported through each source cannot exceed its supply capacity, the requirements of each destination must be satisfied and the total transported amount must not exceed the capacity of vehicles. Again if we carry breakable item on some route then it is required to inflict the rate of breakability on that route, for this motive in this manuscript we inflict the percentage of breakability. In our respective problems safety constraint are imposed for some risk or difficulties in the road. The determination of future transportation planning is generally based on the past record. But the available data from previous experiments are not always precise, often those are imprecise due to uncertainty in ruling, fluctuate financial market, linguistic information, imperfect statistical analysis, insufficient information, etc. For example, transportation cost depends upon fuel price, labor charges, tax charges, etc., each of which are fluctuate time to time. Similarly supply of a source can't be always exact, because it depends upon the availability of manpower, raw-materials, market competition, product demands, etc. Fuzzy set theory and random set theory are most widely used and successfully applied tools to deal with uncertainty. In the next section we formulate four STPs with safety constraints and different uncertain (crisp, fuzzy, hybrid) parameters.

\section{Assumption and Notation}

(i) M: Number of plants in Solid transportation problem.

(ii) N: Number of destinations in Solid transportation problem.

(iii) K: Number of conveyances in Solid transportation problem.

(iv) $\xi_{i j k}=$ Unit transportation cost to transport the commodity from $\mathrm{i}$-th source to $\mathrm{j}$-th destination by $\mathrm{k}$-th conveyances.

(v) $\xi_{i j k}=$ Unknown quantity which is to be transported from i-th source to $\mathrm{j}$-th destination by $\mathrm{k}$-th conveyance(decision variable). plant.

(vi) $a_{i}=$ amount of homogeneous product available at the $\mathrm{i}-\mathrm{th}$

(vii) $b_{j}=$ demand at the $\mathbf{j}$-th destination.

(viii) $e_{k}=$ amount of product which can be carried by the $\mathrm{k}$-th conveyance.

(ix) $\alpha_{i j k}=$ Rate of breaking item of the Solid transportation problem from $\mathrm{i}$-th plant to $\mathrm{j}$-th by $\mathrm{k}$-th conveyance.

(x) $\xi_{i j k}=$ Hybrid unit transportation cost to transport the commodity from $\mathrm{i}$-th source to $\mathrm{j}-\mathrm{th}$ destination by $\mathrm{k}$-th conveyances.

(xi) $\tilde{a}_{i}=$ Fuzzy amount of homogeneous product available at the $\mathrm{i}-$ th plant.

(xii) $\tilde{b}_{j}=$ Fuzzy demand at the $\mathrm{j}$-th destination.

(xiii) $e_{k}=$ Fuzzy amount of product which can be carried by the $\mathrm{k}$-th conveyance.

(xiv) $S_{i j k}=$ the safety factor when an item is transported from the $i$-th plant to $j$-th destination by $k$-th conveyance. (xv) $\widetilde{S}_{i j k}=$ Fuzzy safety factor when an item is transported from the $\mathrm{i}$-th plant to $\mathrm{j}$-th destination by $\mathrm{k}$-th conveyance.

(xvi) $\widehat{\widetilde{S}}_{i j k}=$ Hybrid safety factor when an item is transported from the $\mathrm{i}$-th plant to $\mathrm{j}$-th destination by $\mathrm{k}$-th conveyance.

(xvii) $\mathrm{B}=$ Desired safety measure (DSM) for whole transportation system.

(xviii) $\widehat{\alpha}_{i j k}$ Fuzzy rate of breaking item of the Solid transportation problem from $\mathrm{i}$-th plant to $\mathrm{j}$-th by $\mathrm{k}$-th conveyance.

(xix) $\widetilde{\alpha}_{i j k}$ Hybrid rate of breaking item of the Solid transportation problem from $\mathrm{i}$-th plant to $\mathrm{j}$-th by $\mathrm{k}$-th conveyance.

(xx) If an item is transported from source $i$-th to destination $j$-th by k-th conveyance and then the safety factor $S_{i j k}$ is considered. This implies that if $x_{i j k}>0$, and then we consider the safety factor for this route as a part of the safety constraint. Thus for the convenience of modeling, the following notation is introduced:

$$
Y_{i j k}=\left\{\begin{array}{l}
1 \\
\text { forx }_{i j k}>0 \text { otherwise } \\
0
\end{array}\right.
$$

(xxi) $f_{i j k}=$ fixed charge to transport the commodities from i-th plant to $\mathrm{j}$-th destination by $\mathrm{k}$-th conveyance.

\section{Model Formulation}

According to the above assumption and notation we formulate the following models on transportation problems:

Model-1: Formulation of fixed charge STP with crisp penalties, resources, demands, conveyance capacities and without safety factors, breakability:

After taking all the system parameters and the resources, etc., are deterministic and precisely we formulate the following model as without safety factor and without breakability:

$$
\operatorname{Min}(\mathrm{Z})=\sum_{i=1}^{M} \sum_{j=1}^{N} \sum_{k=1}^{K} \xi_{i j k} x_{i j k}+\sum_{i=1}^{M} \sum_{j=1}^{N} \sum_{k=1}^{K} f_{i j k} y_{i j k}
$$

Subject to the constraints

$$
\begin{aligned}
& \sum_{j=1}^{N} \sum_{k=1}^{K} x_{i j k} \leq a_{i}, i=1,2,3, \ldots \ldots \ldots, \mathrm{M} \\
& \sum_{i=1}^{M} \sum_{k=1}^{K} x_{i j k} \geq b_{i}, j=1,2,3, \ldots \ldots \ldots, N \\
& \sum_{j=1}^{n} \sum_{k=1}^{k} x_{i j k} \leq e_{k}, k=1,2,3, \ldots \ldots \ldots, K \\
& x_{i j k} \geq 0, \forall i, j, k
\end{aligned}
$$

The problem is unbalanced since $\sum_{i=1}^{M} a_{i} \neq \sum_{i=1}^{N} b_{i} \neq \sum_{k=1}^{K} e_{k}$ and has feasible solution if $\sum_{i=1}^{M} a_{i} \geq \sum_{j=1}^{N} b_{j}$ and $\sum_{k=1}^{K} e_{k} \geq \sum_{j=1}^{N} b_{j}$

Model-2: Formulation of STP with crisp penalties, resources, demands, conveyance capacities, breakability and without safety factors:

Considering the entire scheme parameters and the resources, etc., 
are deterministic and precisely we formulate the following model without safety factor and with crisp breakability:

$$
\operatorname{Min}(\mathrm{Z})=\sum_{i=1}^{M} \sum_{j=1}^{N} \sum_{k=1}^{K} \xi_{i j k} x_{i j k}+\sum_{i=1}^{M} \sum_{j=1}^{N} \sum_{k=1}^{K} f_{i j k} y_{i j k}
$$

Subject to the constraints

$$
\begin{aligned}
& \sum_{j=1}^{N} \sum_{k=1}^{K} x_{i j k} \leq a_{i}, i=1,2,3, \ldots \ldots \ldots, M, \\
& \sum_{i=1}^{N} \sum_{k=1}^{K} x_{i j k}\left(1-\alpha_{\mathrm{ijk}}\right) \geq b_{i}, j=1,2, \ldots, N, \\
& \sum_{j=1}^{n} \sum_{k=1}^{k}, x_{i j k} \leq e_{k}, k=1,2, \ldots \ldots, K, \\
& x_{i j k} \geq 0, \forall i, j, k .
\end{aligned}
$$

Model-3: Formulation of STP with crisp penalties, resources, demands, conveyance capacities, safety factors and breakability:

In view of the unit transportation cost, supplies, demands, conveyances capacities, safety factors and breakability as a crisp number we formulate the model as follows:

$$
\operatorname{Min}(\mathrm{Z})=\sum_{i=1}^{m} \sum_{j=1}^{K} \sum_{k=1}^{K} \xi_{i j k} x_{i j k}+\sum_{i=1}^{M} \sum_{j=1}^{N} \sum_{k=1}^{K} f_{i j k} y_{i j k}
$$

Subject to the constraints (5), (7) and

$$
\begin{aligned}
& \sum_{i=1}^{m} \sum_{j=1}^{n} \sum_{k=1}^{K} S_{i j k} y_{i j k} \geq B \\
& \sum_{i=1}^{M} \sum_{k=1}^{K} x_{i j k}\left(1-\alpha_{i j k}\right) \geq \mathrm{b}_{\mathrm{j}}, j=1,2, \ldots, \mathrm{N} \\
& x_{i j k} \geq 0, \forall i, j, k .
\end{aligned}
$$

Model 4: Formulation of STP with hybrid penalties, fuzzy resources, demands, conveyance capacities, breakability and without safety factors.

So in this model formulation we consider the unit transportation cost, resources, demands, conveyance capacities and breakability as fuzzy:

$$
\operatorname{Min}(\mathrm{Z})=\sum_{i=1}^{m} \sum_{j=1}^{n} \sum_{k=1}^{K} \tilde{\xi}_{i j k} x_{i j k}+\sum_{i=1}^{M} \sum_{j=1}^{N} \sum_{k=1}^{K} \tilde{f}_{i j k} y_{i j k}
$$

Subject to the constraints,

$$
\begin{aligned}
& \sum_{j=1}^{N} \sum_{k=1}^{K} x_{i j k} \leq \tilde{a}_{i}, i=1,2, \ldots, \mathrm{M} \\
& \sum_{j=1}^{M} \sum_{k=1}^{K} x_{i j k}\left(1-\tilde{a}_{i j k}\right) \geq \tilde{b}_{j} j=1,2, \ldots, N \\
& \sum_{j=1}^{n} \sum_{k=1}^{K} x_{i j k} \leq \tilde{e}_{k}, k=1,2, \ldots, K \\
& x_{i j k} \geq 0, \forall i, j, k .
\end{aligned}
$$

Model-5: Formulation of STP with hybrid penalties, fuzzy resources, demands, conveyance capacities, safety factors and breakability:

In this model formulation we consider unit transportation cost, resources, demands, conveyance capacities, safety factors and breakability as fuzzy number:

$$
\operatorname{Min}(\mathrm{Z})=\sum_{i=1}^{m} \sum_{j=1}^{n} \sum_{k=1}^{K} \tilde{\xi}_{i j k} x_{i j k}+\sum_{i=1}^{M} \sum_{j=1}^{N} \sum_{k=1}^{K} \widetilde{f}_{i j k} y_{i j k}
$$

Subject to the constraints (11), (13) and

$$
\sum_{i=1}^{m} \sum_{j=1}^{n} \sum_{k=1}^{K} \tilde{S}_{i j k} x_{i j k} \geq \widetilde{B}
$$

$$
\begin{aligned}
& \sum_{i=1}^{M} \sum_{k=1}^{K} x_{i j k}\left(1-\tilde{\alpha}_{i j k}\right) \geq \tilde{\mathrm{b}}_{\mathrm{j}}, j=1,2, \ldots N \\
& x_{i j k} \geq 0, \forall i, j, k .
\end{aligned}
$$

Model-6: Formulation of STP with hybrid penalties, resources, demands, conveyance capacities, safety factors and breakability:

It may happen that the demand or any factor of a commodity in the society is uncertain, not precisely known, but some past data about it is available. From the available records, the probability distribution of demand or any other factor of the commodity can be determined. In a hybrid number we have the combination of fuzziness and randomness. Here we consider the unit transportation cost, resources, demands, conveyance capacities, safety factors and breakability as hybrid number:

$$
\operatorname{Min}(\mathrm{Z})=\sum_{i=1}^{m} \sum_{j=1}^{n} \sum_{k=1}^{K} \tilde{\xi}_{i j k} x_{i j k}+\sum_{i=1}^{M} \sum_{j=1}^{N} \sum_{k=1}^{K} \widetilde{f}_{i j k} y_{i j k}
$$

Subject to the constraint

$$
\begin{aligned}
& \sum_{j=1}^{n} \sum_{k=1}^{k} x_{i j k} \leq \tilde{\hat{a}}_{i}, i=1,2, \ldots, M \\
& \sum_{i=1}^{M} \sum_{k=1}^{K} x_{i j k}\left(1-\tilde{\hat{a}}_{i j k}\right) \geq \tilde{\hat{b}}_{j}, j=1,2, \ldots, N \\
& \sum_{j=1}^{n} \sum_{k=1}^{k} x_{i j k} \leq \hat{\tilde{e}}_{k}, k=1,2, \ldots, K \\
& \sum_{i=1}^{m} \sum_{j=1}^{n} \sum_{k=1}^{k} \hat{\widetilde{S}}_{i j k} Y_{i j k} \geq \widehat{\vec{B}} \\
& x_{i j k} \geq 0, \forall i, j, k .
\end{aligned}
$$

\section{Solution Methodology}

Liu and Liu in 2002 introduced a spectrum of expected value model of fuzzy programming to obtain optimum expected value of objective function $\approx$ under some expected constraints. Considering hybrid penalty $\xi_{i j k}$ are in the form, $\xi_{i j k}=\xi_{i j k}+\xi_{i j k} ; \xi_{i j k}$ where $\xi$ denote the triangular fuzzy number (TFN) and $\hat{\xi}_{i j k}$ is normally distributed random variable with known mean $\bar{\xi}$ and variance $\operatorname{Var}\left(\hat{\xi}_{i j k}\right)$ and constructing expected value model (Liu and Liu 2002; Yang and Liu 2007; Yang and Feng 2007) and using expected value model we have the crisp equivalent of the respective models. The reduced crisp models are solved using constraint optimization software LINGO 13.0 and Genetic Algorithm (GA) (soft computing technique).

Crisp conversion of the Model-4, 5, 6:

For model-4, we have the corresponding crisp form as

$\sum_{i=1}^{m} \sum_{j=1}^{n} \sum_{k=1}^{k} E\left(\tilde{\hat{\xi}}_{i j k}\right) x_{i j k}+\sum_{i=1}^{m} \sum_{j=1}^{n} \sum_{k=1}^{k} E\left(\tilde{\hat{f}}_{i j k}\right) y_{i j k}$

Subject to the constraints,

$$
\begin{aligned}
& \sum_{j=1}^{N} \sum_{k=1}^{k} x_{i j k}<=E\left(\tilde{\mathrm{a}}_{\mathrm{i}}\right), \mathrm{i}=1,2, \ldots, \mathrm{M} \\
& \sum_{i=1}^{M} \sum_{k=1}^{k} x_{i j k}\left(1-E\left(\tilde{\alpha}_{\mathrm{ijk}}\right)\right) \geq E\left(\tilde{\mathrm{b}}_{\mathrm{j}}\right), \mathrm{j}=1, \ldots, \mathrm{N} \\
& \sum_{j=1}^{n} \sum_{k=1}^{k} x_{i j k} \leq \mathrm{E}\left(\tilde{\mathrm{e}}_{\mathrm{k}}\right), \mathrm{k}=1,2, \ldots, \mathrm{K} \\
& \mathrm{x}_{\mathrm{ijk}} \geq 0, \forall i, j, k .
\end{aligned}
$$

For Model-5, we have the corresponding crisp form as 
(20), (21), (22), (23) and

$$
\begin{aligned}
& \sum_{i=1}^{m} \sum_{j=1}^{n} \sum_{k=1}^{k} E\left(\tilde{\mathrm{S}}_{\mathrm{ijk}}\right) y_{i j k}>E(\widetilde{\mathrm{B}}) \\
& \mathrm{x}_{\mathrm{ijk}} \geq 0, \forall i, j, k .
\end{aligned}
$$

For Model-6, we have the corresponding crisp form as, (20) and subject to the constraint

$$
\begin{aligned}
& \sum_{j=1}^{N} \sum_{k=1}^{k} x_{i j k} \leq \mathrm{E}\left(\widetilde{\hat{\mathrm{a}_{\mathrm{i}}}}\right), \mathrm{i}=1,2, \ldots, \mathrm{M} \\
& \sum_{i=1}^{M} \sum_{k=1}^{k} x_{i j k}\left(1-\tilde{\mathrm{E}}\left(\widetilde{\alpha_{\mathrm{ijk}}}\right)\right) \geq \mathrm{E}\left(\tilde{\hat{b}}_{j}\right), \mathrm{j}=1,2, \ldots, \mathrm{N} \\
& \sum_{j=1}^{n} \sum_{k=1}^{k} x_{i j k} \leq \mathrm{E}\left(\hat{\mathrm{e}}_{\mathrm{k}}\right), \mathrm{k}=1,2, \ldots, \mathrm{K} \\
& \sum_{i=1}^{m} \sum_{j=1}^{n} \sum_{k=1}^{k} E\left(\tilde{\hat{\mathrm{S}}}_{\mathrm{ijk}}\right) y_{i j k}>E(\tilde{\mathrm{B}}) \\
& \mathrm{x}_{\mathrm{ijk}} \geq 0, \forall i, j, k .
\end{aligned}
$$

\section{Numerical Experiments}

An item is transported from three plants (at Kolkata, Delhi and Mumbai) to three destinations (at Agartala, Agra and Assam) by three different modes of transport as cargo flight, train and truck. Due to breakability few item as damage.

Sometime the percentage of breakability in the transportation problem, the unit transportation costs, resources, demands at destinations, capacities of the conveyances and safety factor are not known precisely but some past data is available. So it is possible to assume the above as hybrid or fuzzy.

Therefore for model-1, 2, 3 the percentage of breakability in the transportation is 3 and for model- 4,5 and model- 6 the above correspondence are $(2,3,5)$ and $(1,2.3,2.5)+(1.5, .99)$ respectively. The unit transportation costs, resources, demands at destinations, capacities of the conveyances and safety factor (for models 1 to 6) are given below:

\section{Input data}

\section{Crisp Unit transportation costs (in \$):}

$$
\begin{aligned}
& \xi_{111}=18, \xi_{211}=20, \xi_{311}=25, \xi_{121}=26, \\
& \xi_{221}=29, \xi_{321}=24, \xi_{113}=30, \xi_{213}=21, \\
& \xi_{313}=24, \xi_{131}=25, \xi_{231}=23, \xi_{331}=30, \\
& \xi_{112}=22.5, \xi_{212}=21, \xi_{312}=19, \xi_{123}=35, \\
& \xi_{223}=38, \xi_{323}=21.7, \xi_{122}=23.9, \xi_{222}=26.8, \\
& \xi_{322}=32.1, \xi_{132}=23.5, \xi_{232}=22.4, \xi_{332}=37.6, \\
& \xi_{133}=27, \xi_{233}=25.1, \xi_{333}=30.2,
\end{aligned}
$$

\section{Hybrid Unit transportation costs (in \$):}

$$
\begin{aligned}
& \tilde{\hat{\xi}}_{111}=(10,12,13)+(7,0.5), \tilde{\hat{\xi}}_{211}=(8,11,14)+(9,1.7), \\
& \tilde{\hat{\xi}}_{311}=(11,15,17)+(10,2), \tilde{\hat{\xi}}_{121}=(12,14,17)+(11,1.8), \\
& \tilde{\hat{\xi}}_{221}=(13,18,19)+(13,3), \tilde{\hat{\xi}}_{321}=(7,8,11)+(15,1.2),
\end{aligned}
$$

$$
\begin{aligned}
& \tilde{\hat{\xi}}_{113}=(6,7,11)+(22,3), \tilde{\hat{\xi}}_{213}=(7,12,18)+(8,1.35), \\
& \tilde{\hat{\xi}}_{313}=(12,18,22)+(6,1.23), \tilde{\hat{\xi}}_{131}=(16,17,18)+(8,1.44), \\
& \tilde{\hat{\xi}}_{231}=(13,17,18)+(7,3), \tilde{\hat{\xi}}_{331}=(8,17,19)+(15,1.22), \\
& \tilde{\hat{\xi}}_{112}=(11,12,14)+(11,1.2), \tilde{\hat{\xi}}_{212}=(11,12,15)+(9,2), \\
& \tilde{\hat{\xi}}_{312}=(12,13,15)+(6,1), \tilde{\hat{\xi}}_{123}=(14,19,20)+(19,0.85), \\
& \tilde{\hat{\xi}}_{223}=(13,15,19)+(23,2.22), \tilde{\hat{\xi}}_{323}=(15,16,20)+(5,0.27), \\
& \tilde{\hat{\xi}}_{122}=(9,10,11)+(14,2.2), \tilde{\hat{\xi}}_{222}=(10,11,12)+(17,2.3), \\
& \tilde{\hat{\xi}}_{322}=(16,17,19)+(16,1.44), \tilde{\hat{\xi}}_{132}=(14,17,20)+(7,1.9), \\
& \tilde{\hat{\xi}}_{232}=(9,13,18)+(10,0.65), \tilde{\hat{\xi}}_{332}=(17,19,21)+(19,1.75), \\
& \tilde{\hat{\xi}}_{133}=(9,11,12)+(17,1.33), \tilde{\hat{\xi}}_{233}=(10,15,16)+(12,0.95), \tilde{\hat{\xi}}_{333}=(15,19,23)+(11,0.77)
\end{aligned}
$$

\section{Crisp Safety Factors:}

$$
\begin{aligned}
& S_{111}=0.7, S_{211}=0.75, S_{311}=0.85, S_{121}=0.61, \mathrm{~S}_{221}=0.5, \\
& S_{321}=0.57, S_{131}=0.82, S_{231}=0.75, \mathrm{~S}_{331}=0.82, \\
& S_{112}=0.71, S_{212}=0.91, S_{312}=0.52, S_{122}=0.73, \\
& S_{222}=0.79, S_{322}=0.9, S_{132}=0.83, \mathrm{~S}_{232}=0.73, \\
& S_{332}=0.65, S_{113}=0.55, S_{213}=0.74, \mathrm{~S}_{313}=0.86, \\
& S_{123}=0.96, S_{223}=0.68, S_{323}=0.64, \mathrm{~S}_{133}=0.77, \\
& S_{233}=0.78, \mathrm{~S}_{333}=0.59 .
\end{aligned}
$$

\section{Safety Factors as Triangular Fuzzy Number:}

$$
\begin{aligned}
& \widetilde{S}_{111}=(.7, .71, .72), \tilde{\mathrm{S}}_{211}=(.71, .73, .75), \\
& \widetilde{S}_{311}=(.81, .84, .86), \tilde{\mathrm{S}}_{121}=(.55, .59, .63), \\
& \tilde{S}_{221}=(.46, .50, .51), \tilde{\mathrm{S}}_{321}=(.52, .56, .58), \\
& \widetilde{S}_{131}=(.80, .82, .83), \tilde{\mathrm{S}}_{231}=(.72, .75, .78), \\
& \widetilde{S}_{331}=(.81, .82, .83), \tilde{\mathrm{S}}_{112}=(.70, .71, .73), \\
& \widetilde{S}_{212}=(.89, .91, .94), \tilde{\mathrm{S}}_{312}=(.47, .52, .53), \\
& \widetilde{S}_{122}=(.71, .73, .76), \tilde{\mathrm{S}}_{222}=(.76, .79, .82), \\
& \widetilde{S}_{322}=(.87, .92, .93), \tilde{\mathrm{S}}_{132}=(.80, .83, .84), \\
& \widetilde{S}_{232}=(.7, .73, .74), \tilde{\mathrm{S}}_{332}=(.62, .64, .67), \\
& \widetilde{S}_{113}=(.53, .55, .58), \tilde{\mathrm{S}}_{213}=(.73, .74, .78), \\
& \tilde{S}_{313}=(.83, .86, .88), \tilde{\mathrm{S}}_{123}=(.92, .96, .98), \\
& \tilde{S}_{223}=(.64, .68, .69), \tilde{\mathrm{S}}_{323}=(.63, .64, .68), \\
& \tilde{S}_{133}=(.74, .77, .78), \tilde{\mathrm{S}}_{233}=(.75, .78, .80), \\
& \tilde{\mathrm{S}}_{333}=(.55, .59, .62) .
\end{aligned}
$$




\section{Safety Factors as Hybrid variables:}$$
\approx
$$$$
\widehat{S}_{111}=(.45, .46, .47)+(.20, .15),
$$$$
\widetilde{\widehat{S}}_{211}=(.45, .47, .5)+(.30, .43) \text {, }
$$$$
\widetilde{\widehat{S}}_{311}=(.45, .47, .48)+(.40, .25) \text {, }
$$$$
\widetilde{\widehat{S}}_{121}=(.32, .34, .38)+(.20, .41) \text {, }
$$$$
\widetilde{\widehat{S}}_{221}=(.35, .40, .44)+(.1, .25) \text {, }
$$$$
\widetilde{\widehat{S}}_{321}=(.40, .43, .45)+(.17, .32) \text {, }
$$$$
\widetilde{\widehat{S}}_{131}=(.55, .80, .83)+(.22, .32) \text {, }
$$$$
\widetilde{\widehat{S}}_{231}=(.50, .55, .58)+(.25, .34) \text {, }
$$$$
\widetilde{\widehat{S}}_{331}=(.52, .53, .54)+(.35, .3) \text {, }
$$$$
\approx
$$$$
\widehat{S}_{112}=(.40, .41, .42)+(.3, .40) \text {, }
$$$$
\widetilde{\widehat{S}}_{212}=(.53, .54, .55)+(.37, .41) \text {, }
$$$$
\widetilde{\widehat{S}}_{312}=(.29, .30, .33)+(.20, .13) \text {, }
$$$$
\widetilde{\widehat{S}}_{122}=(.41, .43, .45)+(.30, .14) \text {, }
$$$$
\widetilde{\widehat{S}}_{222}=(.37, .39, .4)+(.40, .15) \text {, }
$$$$
\widetilde{\widehat{S}}_{322}=(.51, .53, .54)+(.35, .24) \text {, }
$$$$
\widetilde{\widehat{S}}_{132}=(.45, .46, .47)+(.35, .37) \text {, }
$$$$
\widetilde{\widehat{S}}_{232}=(.3, .33, .34)+(.39, .26) \text {, }
$$$$
\widetilde{\widehat{S}}_{232}=(.3, .33, .34)+(.39, .26) \text {, }
$$$$
\widetilde{\widehat{S}}_{113}=(.22, .25, .28)+(.31, .24) \text {, }
$$$$
\widetilde{\widehat{S}}_{213}=(.34, .35, .38)+(.35, .21) \text {, }
$$$$
\widetilde{\widehat{S}}_{313}=(.52, .56, .59)+(.31, .22) \text {, }
$$$$
\widetilde{\widehat{S}}_{123}=(.61, .66, .68)+(.29, .23) \text {, }
$$$$
\widetilde{\widehat{S}}_{223}=(.31, .32, .35)+(.34, .31) \text {, }
$$$$
\widetilde{\widehat{S}}_{323}=(.41, .44, .45)+(.18, .32) \text {, }
$$$$
\widetilde{\widehat{S}}_{133}=(.34, .37, .38)+(.41, .19) \text {, }
$$$$
\widetilde{\widehat{S}}_{233}=(.45, .48, .52)+(.30, .12) \text {, }
$$$$
\widetilde{\widehat{S}}_{333}=(.33, .39, .40)+(.20, .46) \text {, }
$$

\section{Crisp fixed charge:}

$f_{111}=11.8, f_{211}=12, f_{311}=12.6, f_{221}=12.9$,

$$
\begin{aligned}
& f_{321}=12.4, f_{131}=12.5, f_{231}=12.3, f_{331}=13.0, \\
& f_{112}=10.5, f_{212}=12.1, f_{312}=11.9, f_{122}=12.34, \\
& f_{222}=12.68, f_{322}=13.21, f_{132}=12.35, f_{232}=12.24, \\
& f_{332}=13.76, f_{113}=13.0, f_{213}=12.1, f_{313}=13.0, \\
& f_{123}=13.5, f_{223}=13.8, f_{323}=12.17, f_{133}=12.7, \\
& f_{233}=12.51, f_{333}=13.02 .
\end{aligned}
$$

\section{Hybrid fixed charge:}

$\tilde{f}_{111}=(3,4,5)+(7,0.3), \tilde{\tilde{f}} 211=(4,6,7)+(6,0.5)$

$\tilde{\tilde{f}}_{311}=(5,5.5,7)+(5.8,2), \tilde{\tilde{f}}_{121}=(8,9,10)+(4,0.9)$,

$\tilde{f}_{221}=(8,9,11)+(2,0.5), \tilde{f}_{321}=(10,11,12)+(1,0.5)$,

$\tilde{f}_{131}=(4,6,8)+(7,7.7), \tilde{f}_{231}=(8,9,10)+(3.2,2)$,

$\tilde{\tilde{f}}_{331}=(7,7.8)+(5,0.6), \tilde{f}_{112}=(6,7,8)+(3.25,3)$,

$\tilde{f}_{212}=(3,5,7)+(7.1,3), \tilde{f}_{312}=(5,6,7)+(5.9,2)$,

$\tilde{\tilde{f}}_{122}=(3,4.5,5.5)+(7.34,1), \tilde{\tilde{f}}_{222}=(6,7,8.1)+(5.68)$,

$\tilde{\tilde{f}}_{322}=(6.1,7.9,8)+(6.68,3), \tilde{f}_{132}=(12.35), \tilde{f}_{232}=(6,7.8,8.2)+(4.24, .8), \tilde{\tilde{f}}_{332}=(8,8.9,9.5)+(5.78,2.1)$,

$\tilde{\tilde{f}}_{113}=(6,8,10.4)+(5.6,2)$,

$\tilde{\tilde{f}}_{213}=(2.5,5.7,8.9)+(6,1.2), \tilde{\tilde{f}}_{213}=(10,11,12)+(2,3)$,

$\tilde{\tilde{f}}_{213}=(6.7,7.8,9.8)+(5.6,3), \tilde{f}_{213}=(2.5,5.7,8.9)+(6,1.2)$,

$\tilde{f}_{313}=(10,11,12)+(2,3)$,

$\tilde{\tilde{f}}_{123}=(6.7,7.8,9.8)+(5.6,3)$,

$\tilde{\tilde{f}}_{223}=(10,11.2,12.3)+(2.8,2), \tilde{\tilde{f}}_{323}=(10,10.2,11.3)+(3.8,2.3) 12.17$,

$\tilde{f}_{133}=(4,6,8)+(5.7,4), \tilde{f}_{233}=(8,8.1,8.9)+(3,2.4)$,

$\tilde{\tilde{f}}_{333}=(10,11.4,11.9)+(3.03,1)$.

\section{Crisp Resources, Demands, and Conveyance capacities:}

$a_{1}=64, a_{2}=64, a_{3}=70, b_{1}=41, b_{2}=43, b_{3}=41, e_{1}=45, e_{2}=43, e_{3}=41$

Resources, Demands and Conveyance capacities as a TFN: $\tilde{a}_{1}=(61,64,67), \tilde{\mathrm{a}}_{2}=(62,66,68), \tilde{\mathrm{a}}_{3}=(70,71,72)$,

$\tilde{\mathrm{b}}_{1}=(40,42,43), \tilde{\mathrm{b}}_{2}=(42,43,45), \tilde{\mathrm{b}}_{3}=(43,45,46)$, $\tilde{\mathrm{e}}_{1}=(47,49,50), \tilde{\mathrm{e}}_{2}=(43,46,48), \tilde{\mathrm{e}}_{3}=(41,44,47$.

Resources, Demands and Conveyance capacities as a Hybrid variable:

$\tilde{\hat{a}}_{1}=(51,54,57)+(9,2), \tilde{\hat{a}}_{2}=(52,53,56)+(12,2.3), \tilde{\hat{a}}_{3}=(60,63,64)+(8,5)$, $\tilde{\hat{\mathrm{b}}}_{1}=(35,36,37)+(8,2), \tilde{\hat{\mathrm{b}}}_{2}=(26,29,30)+(16,6), \tilde{\hat{\mathrm{b}}}_{3}=(25,28,29)+(13,2)$, $\tilde{\hat{\mathrm{e}}}_{1}=(27,30,31)+\tilde{\hat{\mathrm{e}}}_{2}=(31,32,34)+(12,7) \tilde{\hat{\mathrm{e}}}_{3}=(28,29,30)+(13 \cdot 9,8)$. 
Citation: Baidya A, Bera UK, Maiti M (2014) Breakable Solid Transportation Problem with Hybrid and Fuzzy Safety Factors using LINGO and Genetic Algorithm. J Appl Computat Math 3: 185. doi:10.4172/2168-9679.1000185

Page 10 of 12

Desired minimum total safety measure for the system:

$$
B=7.72, \widetilde{B}=(7.4,7.5,7.9), \widetilde{\mathrm{B}}=(6.5,6.9,7)+(0.85,1) .
$$

\section{Other parametric values}

Firstly, we set the different parameters on which this GA depends. These are the number of generation (MAXGEN), population size (POPSIZE), probability of crossover (PXOVER), probability of mutation (PMU). There is no clear indication as to how large a population should be. If the population is too large, there may be difficulty in storing the data, but if the population is too small, there may not be enough string for good crossovers. For the present problems, POPSIZE $=100$, PXOVER $=0.7, \mathrm{PMU}=0.3$ and $\mathrm{MAXGEN}=4000$.

\section{Results}

To solve the crisp models we use the LINGO.13 optimization software and GA and the optimal results of the mentioned models are given (Table 1).

\section{Overview of the Results of the Four Models}

The hybrid parameters were first introduced in the literature in 1978, consideration of this type of parameters in the decision making problems is in the developing stage. But, in some real-life problems, available data are hybrid. Hence, decision making problems with hybrid data are of great importance though there are very few such models in the literature. Here for the first time, some constrained unbalanced STPs are formulated with different types of hybrid costs and resources and reduced to corresponding crisp ones using appropriate method. These problems are solved using GA and GRG technique and numerically illustrated. models- 3,5 and 6 are greater than the transportation cost of the models- 2 and 3 since the models 3, 5 and 6 are solved with safety factor as well as breakability but models- 2 and 4 are solved with breakability and without safety factor. The transportation cost of the models with breakability is more than the cost of the other models without breakability because due to breakability the requirement of customer at the end are not fulfill so supplier deliver more quantity as required by customer. Again the transportation cost obtained by generalized reduced gradient technique (LINGO 13.0 Software) is greater than the cost obtained by using Genetic Algorithm. Thus we conclude that to solve any solid transportation problem with and without breakability and safety factor GA is very useful than LINGO (Table 2).

For further research one may apply these techniques for solving interval valued optimization

After solving the respective we observe that the total transportation cost of the model-1 is less than the total transpiration cost of the remaining models since the models- 2 and 4 is solved using breakability and models-3, 5, 6 are solved with breakability and safety factors. Again the total transportation cost of models-3, 5 and 6 are greater than the transportation cost of the models- 2 and 3 since the models 3,5 and 6 are solved with safety factor as well as breakability but models- 2 and 4 are solved with breakability and without safety factor. The transportation cost of the models with breakability is more than the cost of the other models without breakability because due to breakability the requirement of customer at the end are not fulfill so supplier deliver more quantity as required by customer. Again the transportation cost obtained by generalized reduced gradient technique (LINGO 13.0 Software) is greater than the cost obtained by using Genetic Algorithm. Thus we conclude that to solve any solid transportation problem with

\begin{tabular}{|c|c|c|c|c|c|c|}
\hline Optimal & Model-1 & Model-2 & Model-3 & Model-4 & Model-5 & Model-6 \\
\hline \multicolumn{7}{|l|}{ Solution } \\
\hline $\operatorname{Min}(Z)$ & 2848.65 & 2936.37 & 2957.04 & 2817.27 & 2867.02 & 2893.1 \\
\hline$x_{111}$ & 0 & 0 & 0 & 0 & 26.15 & 1.88 \\
\hline$x_{211}$ & 0 & 0 & 0 & 29.6 & 0 & 0 \\
\hline$x_{311}$ & 0 & 2.6 & 0.5 & 0 & 0 & 0 \\
\hline$x_{121}$ & 0 & 0 & 0 & 0 & 4.84 & 8.41 \\
\hline$x_{221}$ & 0 & 0 & 0.62 & 0 & 0 & 0 \\
\hline$x_{321}$ & 0 & 0 & 1.47 & 3.44 & 8.01 & 0.7 \\
\hline$x_{131}$ & 0 & 0 & 31.97 & & 6.1 & 0 \\
\hline$x_{231}$ & 41 & 42.27 & 10.3 & 12.16 & 0 & 34.8 \\
\hline$x_{331}$ & 0 & 0 & 0 & 0 & 0 & 0 \\
\hline$x_{112}$ & 32.66 & 17.94 & 18.45 & 0 & 0.62 & 2.47 \\
\hline$x_{212}$ & 8.34 & 21.73 & 19.4 & 0 & 0 & 13.2 \\
\hline$x_{312}$ & 0 & 0 & 0 & 12.78 & 0 & 17.88 \\
\hline$x_{122}$ & 2 & 3.33 & 5.15 & 0 & 7.95 & 1.918 \\
\hline$x_{222}$ & 0 & 0 & 0 & 0 & 0 & 0 \\
\hline$x_{322}$ & 0 & 0 & 0 & 0 & 0 & 0 \\
\hline$x_{132}$ & 0 & 0 & 0 & 7.97 & 0 & 5.839 \\
\hline$x_{232}$ & 0 & 0 & 0 & 22.24 & 34.43 & 1.45 \\
\hline$x_{332}$ & 0 & 0 & 0 & 0 & 0 & 0 \\
\hline$x_{113}$ & 0 & 0 & 0 & 0 & 0 & 0 \\
\hline$x_{213}$ & 0 & 3.91 & 0 & 0 & 15.608 & 4.7 \\
\hline$x_{313}$ & 0 & 0 & 0 & 0 & 0 & 2.36 \\
\hline$x_{123}$ & 0 & 0 & 0 & 0 & 0 & 0 \\
\hline$x_{223}$ & 0 & 0 & 0 & 0 & 0 & 0 \\
\hline$x_{323}$ & 41 & 41 & 37.08 & 41 & 23.64 & 33.537 \\
\hline$x_{133}$ & 0 & 0 & 0 & 0 & 0.56 & 0 \\
\hline$x_{233}$ & 0 & 0 & 0 & 0 & 0.27 & 0.4 \\
\hline$x_{333}$ & 0 & 0 & 0 & 0 & 0.92 & 0 \\
\hline
\end{tabular}

Table 1: Optimal Results of different Models using LINGO-13.0. 
Citation: Baidya A, Bera UK, Maiti M (2014) Breakable Solid Transportation Problem with Hybrid and Fuzzy Safety Factors using LINGO and Genetic Algorithm. J Appl Computat Math 3: 185. doi:10.4172/2168-9679.1000185

Page 11 of 12

\begin{tabular}{|c|c|c|c|c|c|c|}
\hline Optimal & Model-1 & Model-2 & Model-3 & Model-4 & Model-5 & Model-6 \\
\hline \multicolumn{7}{|l|}{ Solution } \\
\hline $\operatorname{Min}(Z)$ & 2339.2 & 2286.2 & 2343.5 & 2319.3 & 2353.8 & 2155.5 \\
\hline$x_{111}$ & 0 & 0 & 0 & 0 & 0 & 0 \\
\hline$x_{211}$ & 0 & 0 & 0 & 0 & 0 & 0 \\
\hline$x_{311}$ & 33 & 11 & 0 & 1 & 2 & 11 \\
\hline$x_{121}$ & 0 & 0 & 0 & 0 & 0 & 0 \\
\hline$x_{221}$ & 0 & 0 & 0 & 0 & 0 & 0 \\
\hline$x_{321}$ & 0 & 0 & 0 & 31 & 0 & 0 \\
\hline$x_{131}$ & 8 & 0 & 0 & 0 & 0 & 0 \\
\hline$x_{231}$ & 0 & 0 & 0 & 0 & 39 & 21 \\
\hline$x_{331}$ & 0 & 21 & 32 & 0 & 0 & 0 \\
\hline$x_{112}$ & 0 & 0 & 0 & 0 & 6 & 0 \\
\hline$x_{212}$ & 17 & 0 & 0 & 0 & 0 & 0 \\
\hline$x_{312}$ & 0 & 0 & 0 & 0 & 0 & 0 \\
\hline$x_{122}$ & 0 & 0 & 0 & 23 & 14 & 0 \\
\hline$x_{222}$ & 0 & 0 & 0 & 0 & 0 & 0 \\
\hline$x_{322}$ & 0 & 0 & 0 & 0 & 0 & 0 \\
\hline$x_{132}$ & 0 & 23 & 23 & 0 & 17 & 23 \\
\hline$x_{232}$ & 0 & 0 & 0 & 0 & 0 & 0 \\
\hline$x_{332}$ & 0 & 0 & 0 & 0 & 0 & 0 \\
\hline$x_{113}$ & 0 & 0 & 0 & 0 & 0 & 0 \\
\hline$x_{213}$ & 0 & 39 & 39 & 0 & 0 & 39 \\
\hline$x_{313}$ & 0 & 0 & 1 & 0 & 0 & 0 \\
\hline$x_{123}$ & 0 & 0 & 0 & 0 & 0 & 0 \\
\hline$x_{223}$ & 0 & 0 & 0 & 0 & 0 & 1 \\
\hline$x_{323}$ & 0 & 1 & 0 & 1 & 31 & 0 \\
\hline$x_{133}$ & 15 & 0 & 0 & 0 & 0 & 0 \\
\hline$x_{233}$ & 22 & 0 & 0 & 39 & 0 & 0 \\
\hline$x_{333}$ & 0 & 0 & 0 & 0 & 0 & 0 \\
\hline
\end{tabular}

and without breakability and safety factor GA is very useful than LINGO.

For further research one may apply these techniques for solving interval valued optimization problems in the areas of engineering disciplines and management science.

\section{Conclusion}

To prepare this manuscript we consider the unit transportation cost, demand, supplies, conveyances capacity, breakability and safety factor as crisp, fuzzy and hybrid variable. In our manuscript we solve the models- 1 without breakability and safety factors, models- 2 and 3 are solve without safety factor and with breakability and models-3, 5 and 6 are solved with safety factor and breakability. Sometimes impreciseness occurs in transportation for this reason we formulate model-4, 5 and 6 in imprecise environment (fuzzy and hybrid). The result of the respective models as per our expectation i.e., the optimal cost of model-2 and 4 is greater than model- 1 due to breakability and the optimal cost of models-3, 4 and 5 is greater than models- 2 and 4 due to safety factors. The methods, used for solution here are quite general in nature and these can be applied to other similar uncertain/ imprecise models in other areas such as inventory control, ecology, sustainable farm management, etc. In our approach we introduce hybrid transportation cost in solid transportation problem. Finally the entire mathematical models are solved by using LINGO 13.0 software and GA. So our technique is decidedly productive in the wisdom of real life problems of practical importance. Practical numerical examples are provided to demonstrate the feasibility of all decision variables of the proposed methods.

\section{References}

1. Hitchcock FL (1941) The distribution of a product from several sources to numerous localities. Journal of Mathematical Physics 20: 224-230.

2. Shell E (1955) Distribution of a product by several properties. Directorate of Management Analysis. Proceedings of the 2nd Symposium in Linear Programming, DCS/Comptroller HQUSAF Washington, DC, 2: 615-642.

3. Zadeh LA (1965) Fuzzy sets. Information and Control 8: 338-353.

4. Zadeh LA (1978) Fuzzy sets as a basis for a theory of possibility. Fuzzy Sets and Systems 1: 3-28.

5. Kaufmann A (1975) Introduction to the theory of fuzzy subsets, New York: Academic Press

6. Zimmermann HJ (1996) Fuzzy set theory and its applications. Boston: Kluwer Academic Publishers (4th edition).

7. Liu B (2006) A survey of credibility theory. Fuzzy Optimization and Decision Making 5: 387-408.

8. Dubois D, Prade H (1998) Possibility Theory: An Approach to Computerized Processing of Uncertainty. New York: Plenum.

9. Chanas S, Kuchta D (1996) A concept of the optimal solution of the transportation problem with fuzzy cost. Coefficients. Fuzzy Sets and System 82: 299-305.

10. Jimenez F, Verdegay JL (1999) Solving fuzzy Solid transportation problems by an evolutionary algorithm based parametric approach. European Journal of Operational Research 117: 485-510.

11. Liu B, Liu YK (2002) Expected value of fuzzy variable and fuzzy expected value models. IEEE Transactions on Fuzzy Systems 10: 445-450.

12. Yang L, Liu L (2007) Fuzzy fixed charge solid transportation problem and algorithm. Applied Soft Computing 7: 879-889.

13. Chanas S, Kuchta D (1996) Multi-objective programming in optimization of interval objective functions-A generalized approach. European Journal of Operational Research 94: 594-598. 
Citation: Baidya A, Bera UK, Maiti M (2014) Breakable Solid Transportation Problem with Hybrid and Fuzzy Safety Factors using LINGO and Genetic Algorithm. J Appl Computat Math 3: 185. doi:10.4172/2168-9679.1000185

14. Saad OM, Abass SA (2002) A Parametric study on transportation problem under fuzzy environment. Engineering Journal of the University of Qatar 15: 165-176.

15. Grzegorzewski P, Mrowka E (2007) Trapezoidal approximation of fuzzy number-revisited. Fuzzy Sets and Systems 158: 757-768.

16. Chanas S (2001) On the interval approximation of a fuzzy number. Fuzzy Sets and System 122: 353-356.

17. Grzegorzewski P (2002) Nearest interval approximation of a fuzzy number. Fuzzy Sets and Systems 130: 321-330.

18. Kundu P, Kar S, Maiti M (2013) Multi-objective solid transportation problem with budget constraint in uncertain environment. International Journal of System Science.
19. Baidya A, Bera UK, Maiti M (2013) Multi-item interval valued solid transportation problem with safety measure under fuzzy-stochastic environment. Journal of Transportation Security 6: 151-174.

20. Kennington JL, Unger VE (1976) A new branch and bound algorithm for the fixed charge transportation problem. Management Science 22: 1116-1126

21. Sun M, Aronson JE, Mckeown PG, Dennis D (1998) A tabu search heuristic procedure for fixed charge transportation problem. European Journal of Operational Research 106: 411-456.

22. Gottlieb J, PaulmannL (1998) Genetic algorithms for the fixed charge transportation problems. Proceedings of the IEEE Conference on Evolutionary Computation. ICEC 330-335.

23. Holland HJ (1975) Adaptation in natural and artificial systems. University of Michigan. 